\title{
Deposition of Cell-Laden Hydrogels in a Complex Geometry Using a 3D BioPrinter
}

\author{
Sarah Grace Dennis ${ }^{1}$ and Michael J. Yost ${ }^{1}$
}

\section{Medical University of South Carolina, Department of Surgery, Charleston, USA}

Solid freeform fabrication (SFF) corresponds to a variety of technologies capable of additive layer-bylayer manufacture of three-dimensional constructs through computer-aided design (CAD) and computeraided manufacturing (CAM) [1]. Combining SFF and tissue engineering techniques resulted in cell bioprinting. In bioprinting techniques; cells and other biological materials are incorporated into carrier materials during the fabrication process and concurrently extruded using a dispensing device [2-3]. An advantage of this technology is the ability to incorporate three-dimensional medical technologies, such as magnetic resonance imaging (MRI) and computer tomography (CT), into the designs resulting in patient-specific scaffolds [2]. The Palmetto Printer was designed to deposit biomimetic tissues for tissue engineering. This study demonstrates the bioprinter is capable of producing cell-laden hydrogel constructs with a complex geometry while maintaining high cell viability.

Normal human dermal fibroblasts (NHDF's) and human adipose microvascular endothelial cells (HAMEC's) were cultured in FGM-2 and EGM-2, respectively, until confluent. Alginate, the hydrogel, was dissolved in sterile, distilled water to produce a solution with an alginate concentration of $3 \%$. This solution was then autoclaved for sterilization. NHDF and HAMEC cells were suspended in the alginate at a ratio of 4:1 and a concentration of 1.0 million cells per milliliter alginate [4]. The printing substrate was a $100 \mathrm{mM} \mathrm{CaCl}_{2}$ gelatin solution comprising of calcium chloride dehydrate, sodium chloride $(0.9$ wt\%), porcine gelatin (3 wt\%), and titanium dioxide (1 wt\%) in distilled water.

The cell-laden alginate was deposited in a bifurcated ellipse pattern to demonstrate the bioprinter's ability to generate viable structures. The constructs gelled for 10 minutes post-printing. They were then stained, following the kit protocol, with a mammalian viability/cytotoxicity assay by Invitrogen Life Technologies. The stained constructs were imaged one hour post-printing with a Leica TCS SP5 AOBS Confocal Microscope System using Z-stack parameters of X over Y depth. Cells that appear green or yellow are counted as live. Red cells are counted as dead. Cell viability was calculated using the following equation: Viability $=\frac{\# \text { of live cells }}{\# \text { total cells }} \times 100 \%$. Cell viability for the bioprinted ellipses was quantified by imaging five different sections, counting the live and dead cells. Cell viability was 98.1\%.

Additive manufacturing is the logical next step in tissue engineering and design. For tissue engineering, the unique features of the ability to precisely place nanoliter size aliquots of cell containing hydrogel as well as the ability to recapitulate a more natural process will move the field forward substantially. The Palmetto Printer is a step along the way of creating workable solid free form fabrication of living tissues as has been demonstrated here.

1. Ferris, C.J., Gilmore, K.G., Wallace, G.G., \& Panhuis, M. Biofabrication: An Overview of the Approaches Used for Printing of Living Cells. Appl. Microbiol. Biotechnol. 97 (10), 4243-4258, doi: 10.1007/s00253-013-4853-6 (2013).

2. Khalil, S., Nam, J., and Sun, W. Multi-Nozzle Deposition for Construction of 3D Biopolymer Tissue Scaffolds. Rapid Prototyping Journal. 11 (1), 9-17, doi: 10.1108/13552540510573347 (2005). 
3. Blaeser, A., Duarte Campes, D.F., Weber, M., Neuss, S., Theek, B., Fischer, H., and JahnenDechent, W. Biofabrication Under Fluorocarbon: A Novel Freeform Fabrication Technique to Generate High Aspect Ratio Tissue-Engineered Constructs. BioResearch. 2 (5), 374-384, doi: 10.1089/biores.2013.0031 (2013).

4. Czjaka, C.A., Mehesz, A.N., Trusk, T.C., Yost, M.J., and Drake, C.J. Scaffold-Free Tissue Engineering: Organization of the Tissue Cytoskeleton and Its Effects on Tissue Shape. Annals of Biomedical Engineering. 42 (5), 1049-1061, doi: 10.1007/s10439-014-0986-8 (2014).

5. The authors acknowledge funding from the National Science foundation EPS-0903795 and the National Institutes of Health NIDCR R01- DE019355 (MJY PI).
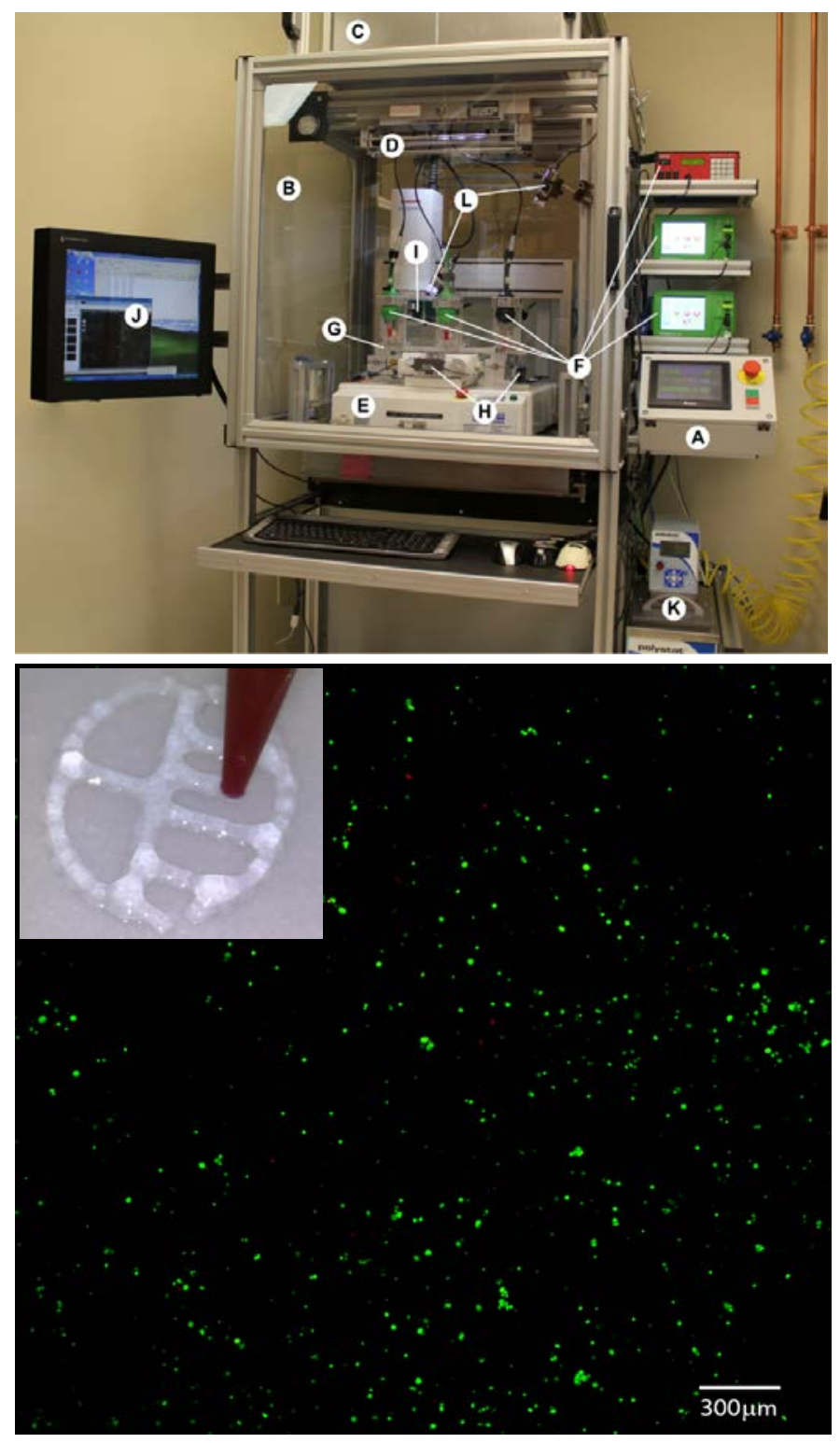

Figure 1: Palmetto Printer. A Programmable Logic Controller (PLC) coordinates the actions of all printer functions (A). The bioprinter is enclosed in an airtight containment chamber (B) with filtered intake and exhaust $(\mathrm{C})$ that maintains a regulated internal positive pressure to reduce risk of contamination. There are dual UV lights (D) that are mounted at the top of the chamber and are controlled by the PLC. The Janome $2300 \mathrm{~N}$ XYZ Robot (E) is programmed and controlled by an integrated computer $(\mathrm{J})$. There is a distance laser (I) mounted on the robotic arm's Z-axis dispenser holder. There are three independent dispenser controllers (F) that are programmed manually to regulate the output of the dispenser guns available to be loaded onto the $\mathrm{Z}$ axis arm under computer control. Temperature of the substrate (printing surface) is maintained by a water bath controller $(\mathrm{K})$ between $4^{\circ} \mathrm{C}$ and $40^{\circ} \mathrm{C}$. Dual digital cameras (L) are available to monitor printer activity and sample formation.

Figure 2: Bioprinted Construct. Image of bioprinted constructs stained with mammalian live/dead fluorescence. Live cells appear green and dead cells appear red taken with Leica TCS SP5 AOBS Confocal Microscope. Inset, photomicrograph of printed ellipse. 